\title{
Some clinical features of liver cell failure: an appraisal of their causes
}

\author{
A. E. READ \\ From the University of Bristol
}

SUMMARY The mechanisms underlying the cause of the major clinical features of liver cell failure are reviewed. These include jaundice, fluid retention, hepatic encephalopathy, bleeding tendency, etc.

New knowledge has highlighted the various metabolic disturbances which result from liver cell disease, both acute and chronic. It is the purpose of this brief review article to examine the causes of some of the clinical features of the syndrome of liver cell failure in the light of these new developments. For this purpose the clinical features are set out in the Table.

\section{Impaired liver cell function}

\section{JAUNDICE}

The patient with cirrhosis may well be icteric and the degree of icterus rapidly deepens if liver cell failure worsens. Haemolysis is one factor increasing bile pigment load and is either an acute syndrome associated with changes in the serum lipids or a chronic one, probably due to sequestration of cells in a hypertrophied spleen and reticuloendothelial system (Zieve, 1966). An increased pigment load might also result from the impaired coagulability of blood with subsequent haemorrhage. The uptake of bilirubin into the hepatocyte is also diminished in chronic liver disease (Barrett et al., 1968). This could involve a disturbance in the inducible acceptor protein Y (ligandin, GSH transferase) and the protein $Z$ found in liver and other tissues (Levi et al., 1969). These seem responsible for the entry of organic anions (such as bilirubin) into the liver and, though the affinities of bilirubin for ligandin and $Z$ are similar, the former system has a greater capacity and is the more important in man.

The conjugation of bilirubin may also be impaired within the liver cell in cirrhosis, though levels of bilirubin UDP glucuronyl transferase in biopsy specimens from patients with cirrhosis are usually normal-except, that is, in the cirrhosis of Wilson's disease (Black and Billing, 1969). However, food deprivation is a known factor which alters bilirubin conjugation and nutritional problems are important
Table Some clinical results of impaired liver cell function

Jaundice

Fluid retention

Hepatic encephalopathy

A bleeding tendency

Clinical results of the altered metabolism of drugs

Diabetes

Endocrine disorders

in liver disease and could produce significant changes.

The manner in which bilirubin conjugates are secreted into the biliary canaliculus is uncertain. Though probably not sharing a metabolic pathway with bile salts, bile acids do have an enhancing effect on biliary flow and about $50 \%$ of bile flow in man is bile salt dependant. Bile salt production is impaired in patients with parenchymal liver disease (Vlahcevic et al., 1971) and this, together with the anatomical derangement associated with cirrhosis, may be the major factors determining the cholestatic features of the jaundice in some cirrhotic patients (Datta et al., 1963).

\section{FLUID RETENTION}

Oedema and ascites are well recognised features of liver cell failure. They may develop acutely when a sudden metabolic insult, such as the effects of intestinal haemorrhage, falls on the compromised (cirrhotic) liver. This type clears rapidly if the stimulus is removed-for example, cessation of bleeding. Fluid retention may, however, be chronic as part of progressive liver cell failure and then even very energetic therapy with diuretics may not be successful in removing it. The aetiology of the fluid retention involves reduced synthesis of albumin by the liver. Albumin synthesis in normal man is not apparently a function of every hepatocyte (Feldmann et al., 1972). Further, in cirrhosis, hypoalbuminaemia is not always associated with 
impaired hepatic albumin synthesis and a normal albumin pool may be demonstrated with increased albumin being found outside the vascular compartment (Rothschild et al, 1969). A distribution abnormality is therefore a fundamental part of the hypoalbuminaemia which, together with a disturbance of the Starling equilibrium, is usually invoked to explain the retention of fluid in the tissues and peritoneum.

Portal hypertension is the major factor that localises fluid retention in the cirrhotic to the abdominal cavity as ascites. In patients with extrahepatic portal hypertension, the raised intracapillary pressure is contained within the splanchnic circulationascites, if it occurs, is of low protein content. In patients with cirrhosis and ascites, portal hypertension is at the level of the hepatic sinusoids and an important factor in its production is hepatic venous outflow block caused by regeneration nodules. The raised intrasinusoidal pressure readily produces ascites due to splanchnic transudation, but, more importantly, because of the production of increased amounts of hepatic lymph which are drained from the liver by lymphatics. When this lymphatic transport route is overwhelmed, lymph pours from the liver surface directly into the abdominal cavity. Theoretically, because hepatic lymph contains a high protein content, ascitic fluid in cirrhotics should have a similar content. In practice, it usually does not, and one must presume that this effect is counterbalanced because of concomitant exudation of more splanchnic protein-poor fluid or by reduction of the protein content of hepatic lymph by changes in the sinusoidal microvasculature (Schaffner and Popper, 1963). This dual origin of ascitic fluid in patients with hepatic cirrhosis explains the recognised superiority of side-to-side rather than end-to-side portocaval shunts in the control of ascites. The former decreases both sinusoidal and splanchnic transudation of fluid and the later only the splanchnic portion.

Though hypoalbuminaemia and portal hypertension seem the major factors which determine the production of ascites, its persistence must be due to other factors, most noticeably those involving renal sodium excretion. These include the increased activity of aldosterone (due to impaired hepatic metabolism), and a failure of the cirrhotic kidney to break through the resultant excessive renal tubular absorption of sodium by exhibiting a (normal) third factor response. The nature of this protective third factor (de Wardener et al., 1961) is unknown. It may well not be a natriuretic chemical in its own right but may act by altering existing regulatory mechanisms, including peritubular hydrostatic and osmotic pressure. Changes in renal vascular perfusion are further ways in which escape from avid sodium restriction may be hindered. Changes in the distribution of the renal circulation have been demonstrated by techniques using radio Xenon (Kew et al., 1971), though these techniques have been shown to possess definite shortcomings. Increasing medullary renal blood flow at the expense of that supplying the cortex might well increase sodium reabsorption by shunting blood to the deeper rather than the more superficial nephrons, where longer loops of Henle might produce more avid sodium retention.

The patient with ascites and peripheral oedema associated with liver disease may well domonstrate, during rapid diuresis, a compartmentalisation effect such that peripheral oedema is more rapidly mobilised than ascites (Shear et al., 1970). This is due to the fixed and slower rate of ascites reabsorption-of about $1 \mathrm{l} /$ day. Increased diuresis in these circumstances is therefore obtained only by excreting peripheral oedema fluid or, if this is not available, fluid from the vascular compartment-with consequent dire effects on renal and hepatic perfusion.

\section{HEPATIC ENCEPHALOPATHY}

Apart from newer theories relating to the cause or causes of this syndrome, it is also recognised that the symptoms and physical signs can be protean and related to dysfunction of many parts of the brainparticularly the cerebellum, basal ganglia, and cortex as well as the spinal cord (Read et al., 1967). These abnormalities are likely to be a feature of 'successful' shunt surgery, where lengthy postoperative survival due to good liver cell function has been a feature.

New theories related to the cause of hepatic encephalopathy have in particular been concerned with an upset in the balance of intracerebral neurotransmitter amines. Two groups of substances have come under scrutiny-namely, false neurotransmitter amines (such as octopamine) and their precursor aromatic amino acids phenylalanine, tyrosine, and tryptophan. In the experimental animal and in patients with hepatic encephalopathy, an increase in brain (Lam et al., 1973) and urinary and blood octopamine has already been noted (Manghani et al., 1975). The beneficial response of some patients with hepatic encephalopathy to L-dopa was also thought to represent the results of 'normalisation' of brain neuroamines (Fischer.and James, 1971).

Octopamine and perhaps other amines produced in the gut and transported to the brain are postulated to act as false neurotransmitters - that is, they resemble structurally normal transmitter neuroamines, but are inactive when fixed at brain synapses. They could certainly act as agents which might seriously alter the local concentration of cerebral neuroamines, perhaps depleting 'alerting' amines such as noradrenaline and dopamine. There is, however, 
grave doubt whether the depletion of brain noradrenaline and dopamine alone could cause hepatic encephalopathy. Certainly, massive experimental reduction of cerebral noradrenaline and dopamine produced in turn by massive intracerebral infusions of octopamine do not cause hepatic encephalopathy (Zieve and Olsen, 1977). These studies do not invalidate, however, the known reduction of brain noradrenaline that occurs in rats in acute hepatic coma, produced by hepatic artery ligation (Dodsworth et al., 1974), but make neuroamine depletion per se an unlikely contender as a cause of hepatic encephalopathy.

Interest developed in the possible relationship of altered serum amino acid profiles to hepatic encephalopathy because certain neutral amino acids, the aromatic amino acids, phenylalanine, tyrosine, and tryptophan are the precursors of the neurotransmitter amines noradrenaline, dopamine, and serotonin, as well as false neurotransmitters such as octopamine. Further, the plasma aminogram of patients with acute hepatic necrosis and chronic liver disease is abnormal (Fischer et al., 1975). A high ratio of aromatic to branch chain amino acids is found particularly in the latter condition, though the aetiology of the hepatic encephalopathy seems important too (Rosen et al., 1977). One factor potentiating this high ratio, and thus perhaps related to the presence of encephalopathy, is the action of insulin. Severe impairment of liver function results in hyperinsulinaemia. The infusion of large amounts of intravenous glucose as part of the therapy of hepatic encephalopathy could of course accent this hyperinsulinaemia and its effects. Insulin promotes the shunting of branched chain amino acids such as leucine, isoleucine, and valine into muscle and therefore competition between branched and aromatic amino acids for entry into the brain is shifted towards the aromatic group with potentiation of neuroamine production (Munro et al., 1975). If this also particularly encouraged the production of cerebral serotonin for which tryptophan is a precursor, hepatic coma might be the result. Though encouraging clinical results following the 'normalisation' of amino acid patterns in the plasma of patients with encephalopathy have been described (Fischer et al., 1976), these seem not to have been widely substantiated.

Other substances perhaps implicated in the production of hepatic encephalopathy include the medium chain fatty acids (Zieve and Nicoloff, 1974). Studies where these have been administered to patients with hepatic encephalopathy have not shown any significant clinical or EEG deterioration (Morgan et al., 1974) and it seems that they are ancillary rather than primary factors, though in large doses their narcotic effect in various animal species is not in doubt (Muto et al., 1964).

\section{BLEEDING TENDENCY}

Disturbances of blood coagulation are common features of liver cell failure and the most plausible explanation is a lack of the coagulant factors which are synthesised in the liver. Thus vitamin $K$ is essential for the activation of four coagulant factors II, VII, IX, and X. Factor I (fibrinogen) and factor $V$ are also synthesised in the liver, but not under the control of vitamin $\mathrm{K}$. The absorption of vitamin $\mathrm{K}$ in liver disease is often impaired, because of reduced bile salt concentration in the gut. This may be aggravated by oral antibiotics such as neomycin. The luminal deficiency results both from biliary obstruction and impaired liver cell synthesis. In the absence of vitamin $\mathrm{K}$ a series of precursor substances are synthesised. In obstructive jaundice it appears that these proteins may also be structurally abnormal (Preston et al., 1977). Factor VIII deficiency is not a prominent feature in liver disease and often, in fulminant liver failure, levels are raised. Perhaps this is related to its synthesis in sites outside the liver (Deutsch, 1965). Similarly, apart from fulminant liver failure, hypofibrinogenaemia is also unusual, except, that is, that due to secondary disseminated intravascular coagulation (DIC). On the other hand, factor $\mathrm{V}$ deficiency is a common finding in patients with disturbed coagulation, secondary to chronic liver disease, and can be effectively treated only by fresh frozen plasma. Although studies in acute hepatic necrosis have shown that diffuse intravascular coagulation does occur (Rake et al., 1970), presumably due to the release of thromboplastins from damaged liver cells, in patients with cirrhosis this mechanism seems less important and, when it does occur, appears to be correlated more with the severity of portal hypertension.

Platelet dysfunction is another important cause of a coagulation disturbance in liver disease-platelet numbers in the peripheral blood are diminished either by the effects of toxins acting directly on the bone marrow-for example, alcohol, folate deficiency (Cowan and Hines 1971) - and also by splenic sequestration, by DIC, and occasionally by an autoimmune mechanism (Habib and Burningham, 1973). Platelet aggregation in response to ADP (Thomas et al., 1967) is reduced in alcoholic cirrhotics and, at least in acute liver cell failure, this abnormality is seen with changes in platelet ultrastructure-particularly an increased content of microtubules (Rubin et al., 1977).

The part played by disturbances of the fibrinolytic system in the production of coagulation disturbances is also uncertain. Increased fibrinolysis is 
found in liver disease and, though this was originally thought to be a primary event, it seems more likely that it is an accompaniment of DIC. Determinations of fibrinopeptide A may enable one to distinguish between 'primary' and 'secondary' fibrinolysis due to DIC as it is relased from fibrinogen only by thrombin - that is, in the secondary form (Nossel et al., 1974). Fibrinolytic activity is, however, decreased in biliary obstruction and was associated with decreased levels of plasminogen activator activity in the serum, in an investigation by Jedrychowski et al., 1973). These authors speculated that inhibition of fibrinolysis might be due to altered lipid metabolism--and that the patient with biliary obstruction undergoing surgery might run the risk of increased thrombosis.

The association between the Dubin Johnson syndrome and isolated factor VII deficiency-found in patients with this condition in Israel-deserves mention. Bleeding was found in about $20 \%$ and a prolonged prothrombin time in $60 \%$ which could not always be corrected by vitamin $\mathrm{K}$. This abnormality, either singly or in combination with the Dubin Johnson syndrome, was also seen in relatives of the cases studied (Seligsohn et al., 1970).

\section{ALTERED DRUG METABOLISM}

Patients with liver disease are clinically unduly sensitive to a number of drugs. This is particularly important with sedative drugs where latent hepatic encephalopathy may be exacerbated. This sensitivity to drugs could presumably result from impaired drug breakdown - a function of the mixed oxidative drug-metabolising enzymes found in hepatocyte smooth endoplasmia reticulum. Though it is an obvious explanation of such a phenomenon in liver disease, it was not until 1968 that proof of diminished drug metabolising capacity became conclusive (Levi et al., 1968), because previous studies had disregarded concurrent drug-induced microsomal enzyme induction. Chlorpromazine is a typical example of a drug with sedative properties that are much accentuated in the presence of liver disease (Read et al., 1969), though drug levels are not abnormally high and increased brain sensitivity rather than impaired metabolism seems an important cause of sensitivity (Maxwell et al., 1972).

Recent studies with diazepam have reinforced these ideas. The amount of drug required to produce a clinical end point (ptosis-nystagmus, dysarthria, and sedation) was much reduced in cirrhotic patients compared with normal subjects, yet there was a good correlation with liver synthetic function as measured by the serum albumin (Branch et al., 1976). The drug levels of diazepam at the conclusion of this study in normal subjects and cirrhotic patients were similar, despite a smaller dose in the cirrhotic patients. This suggests that volume of distribution enlarges with increasing dose. Increased cerebral sensitivity was demonstrated by the increased slowing of the EEG in the cirrhotic as opposed to the control group.

Factors other than rates of metabolism and target organ sensitivity are obviously important when considering drug action in liver disease. Drug elimination may be impaired in the case of agents secreted into bile by anatomical factors and deficient action of the pump responsible for excretion of ionic and non-ionic agents. The first pass effect may be altered by liver cell disease. Volume of distribution may be abnormal, particularly in those with fluid retention and where there is tissue wasting. Important changes in the serum albumin level may also effect plasma binding. Where the albumin level is diminished because of liver cell dysfunction, concentrations of free/bound drug may be increased. The free unbound drug is that part which is clinically active. A low serum albumin may thus cause an apparent increase in therapeutic effect from a fixed dose of a drug which, with a higher serum albumin level, would give a lesser effect. This has certainly been demonstrated with drugs such as prednisolone (Powell and Axelsen, 1972).

\section{DIABETES}

Diabetes is a common feature in chronic liver cell failure associated with cirrhosis. Figures show that up to one-third of cirrhotics have frank diabetes and the majority (up to $80 \%$ ) have impaired glucose tolerance as defined by a glucose tolerance curve (Conn et al., 1969; 1971). Because insulin levels are raised in cirrhotic diabetes (Megyesi et al., 1967), a condition of insulin resistance rather than insulin lack seemed the obvious cause. Glucagon is one obvious hormonal antagonist to insulin which might be associated with this disorder and hyperglucagonaemia is a feature of cirrhosis with portosystemic shunting (Sherwin et al., 1974). The cause of this rise, which is not associated with increased degradation, is unknown.

The nature of the hyperinsulinaemia accompanying cirrhotic diabetes has recently been studied (Johnston et al., 1977). Previously it was uncertain whether this was due to increased pancreatic secretion or diminished hepatic degradation. These workers have compared insulin and $\mathrm{C}$ peptide levels in cirrhotics both when fasting and after glucose loads. The ratio of $\mathrm{C}$ peptide/insulin was decreased in hyperinsulinaemic cirrhotic subjects compared with control subjects and after oral glucose C peptide levels were the same in normoinsulinaemic and hyperinsulinaemic subjects but insulin levels were much increased in the latter group. As the breakdown of proinsulin yields insulin and C 
peptide in equimolar amounts, this suggest that the hyperinsulinaemia of cirrhosis is due to escape of insulin to the periphery, thus escaping its normal hepatic degradation. The 'escape' could therefore be caused by diminished liver cell removal, by portosystemic shunting, or by both factors.

\section{ENDOCRINE DISORDERS}

The clinical picture of 'feminisation' of the male cirrhotic with testicular atrophy, impotence, gynaecomastia, sparse body hair, female body habitus, and reduced prostate size has been recognised for many years. Its cause until recently had been ascribed to abnormal hepatic oestrogen metabolism (Edmondson et al., 1939). However, studies of oestradiol metabolism in cirrhosis have shown normal rates of metabolism (Baker et al., 1976) and, though it is possible that other oestrogenic compounds may be involved, this had led to a reassessment and extension of the search for other possible endocrine causes of this syndrome.

The major findings have been as follows: firstly, there is a significant reduction in total blood testosterone level (Kley et al., 1975) and an even greater fall in that part which is biologically active. These changes are related directly to the testicular atrophy. Secondly, though there is still some confusion, it seems that oestradiol (the most potent oestrogen biologically) shows no major change either in the level of its total or free forms, though the weaker precursor, oestrone, is raised (Green et al., 1976). Because of a failure to find conclusive evidence of hyperoestrogenism, other aspects of oestrogen metabolism, particularly increased peripheral conversion of androgens to oestrogens, have been noted (Vermeulen et al., 1973). Changes in hypothalamic pituitary function have also been defined and, though plasma levels of gonadatrophins are normal despite testicular atrophy, there is a reduced gonadotrophin release (Van Thiel et al., 1974) after clomiphene administration. This is at a hypothalamic rather than pituitary level. How these changes came about is uncertain, and whether the testicular defect itself is likely to be the principal abnormality is far from clear.

A further addition to the endocrine changes in liver disease is the recent description of alcoholinduced pseudo-Cushing's syndrome (Smals et al., 1976; Rees et al., 1977). High corticosteroid levels fall to normal when alcohol is withdrawn.

\section{References}

Baker, H. W. G., Burger, H. G., de Kretser, D. M., Dulmanis, A., Hudson, B., O'Connor, S., Paulsen, C. A., Purcell, N., Rennie, G. C., Seah, C. S., Taft, H. P., and Wang, C. (1976). A study of the endocrine manifestations of hepatic cirrhosis. Quarterly Journal of Medicine, 45, 145-178.

Barrett, P. V. D., Berk, P. D., Menken, M., and Berlin, M. I. (1968). Bilirubin turnover studies in normal and pathologic states using bilirubin ${ }^{14} \mathrm{C}$. Annals of Internal Medicine, 68, 355-377.

Black, M., and Billing, B. H. (1969). Hepatic bilirubin UDP-glucuronyl transferase activity in liver disease and Gilbert's syndrome. New England Journal of Medicine, 280, 1266-1271.

Branch, R. A., Morgan, M. H., James, J., and Read, A. E. (1976). Intravenous administration of diazepam in patients with chronic liver disease. Gut, 17, 975-983.

Conn, H. O., Schreiber, W., and Elkington, S. G. (1971). Cirrhosis and diabetes II. Association of impaired glucose tolerance with portal-systemic shunting in Laennec's cirrhosis. American Journal of Digestive Diseases, 16, 227-239.

Conn, H. O., Schreiber, W., Elkington, S. G., and Johnson, T. R. (1969). Cirrhosis and diabetes I. Increased incidence of diabetes in patients with Laennec's cirrhosis. American Journal of Digestive Diseases, 14, 837-852.

Cowan, D. H., and Hines, J. D. (1971). Thrombocytopenia of severe alcoholism. Annals of Internal Medicine, 74, 37-43.

Datta, D. V., Sherlock, S., and Scheuer, P. J. (1963). Postnecrotic cirrhosis with chronic cholestasis. Gut, 4, 223-230.

Deutsch, E. (1965). Blood coagulation changes in liver diseases. Progress in Liver Diseases, 2, 69-83.

de Wardener, H. E., Mills, I. H., Clapham, W. F., and Hayter, C. J. (1961). Studies on the efferent mechanism of the sodium diuresis which follows the administration of intravenous saline in the dog. Clinical Science, 21, 249-258.

Dodsworth, J. M., James, J. H., Cummings, M. C., and Fischer, J. E. (1974). Depletion of brain norepinephrine in acute hepatic coma. Surgery, 75, 811-820.

Edmondson, H. A., Glass, S. J., and Soll, S. N. (1939). Gynaecomastia associated with cirrhosis of the liver. Proceedings of the Society of Experimental Biology and Medicine, 42, 97-99.

Feldmann, G., Penaud, J., and Crassous, J. (1972). Albumin synthesis by the human liver: its morphologic demonstration. Proceedings of the Vth Meeting of the International Association for the Study of the Liver. Versailles, p. 28.

Fischer, J. E., and James, J. H. (1971). Mechanism of action of L-dopa in hepatic coma. Surgical Forum, 22, 347-349.

Fischer, J. E., Funovics, J. M., Aguirre, A., James, J. H., Keane, J. M., Wesdorp, R. I. C., Yoshimura, N., and Westman, T. (1975). The role of plasma amino acids in hepatic encephalopathy. Surgery, 78, 276-290.

Fischer, J. E., Rosen, H. M., Ebeid, A. M., James, J. H., Keane, J. M., and Soeters, P. B. (1976). The effect of normalization of plasma amino acids on hepatic encephalopathy in man. Surgery, 80, 77-91.

Green, J. R. B., Mowat, N. A. G., Fisher, R. A., and Anderson, D. C. (1976). Plasma oestrogens in ${ }^{\circ}$ men with chronic liver disease. Gut, 17, 426-430.

Habib, M. A., and Burningham, R. A. (1973). Autoimmune thrombocytopenia occurring in a young woman with idiopathic haemochromatosis. Scandinavian Journal of Haematology, 10, 148-152.

Jedrychowski, A., Hillenbrand, P., Ajdukiewicz, A. B., Parbhoo, S. P., and Sherlock, S. (1973). Fibrinolysis in cholestatic jaundice. British Medical Journal, 1, 640-642.

Johnston, D. G., Alberti, K. G. M. M., Faber, O. K., Binder, C., and Wright, R. (1977). Hyperinsulinism of hepatic cirrhosis: diminished degradation or hypersecretion? Lancet, 1, 10-13.

Kew, M. C., Brunt, P. W., Varma, R. R., Hourigan, K. J., Williams, H. S., and Sherlock, S. (1971). Renal and intrarenal blood-flow in cirrhosis of the liver. Lancet, 2 , 
504-510.

Kley, H. K., Nieschalg, E., Wiegelmann, W., Solbach, H. G., and Kruskemper, H. L. (1975). Steroid hormones and their binding in plasma of male patients with fatty liver, chronic hepatitis and cirrhosis. Acta Endocrinologica, 79, 275-285.

Lam, K. C., Tall, A. R., Goldstein, G. B., and Mistilis, S. P. (1973). Role of a false neurotransmitter, octopamine, in the pathogenesis of hepatic and renal encephalopathy. Scandinavian Journal of Gastroenterology, 8, 465-472.

Levi, A. J., Sherlock, S., and Walker, D. (1968). Phenylbutazone and isoniazid metabolism in patients with liver disease in relation to previous drug therapy. Lancet, 1 , 1275-1279.

Levi, A. J., Gatmaitan, Z., and Arias, I. M. (1969). Two hepatic cytoplasmic protein fractions, $Y$ and $Z$, and their possible role in the hepatic-uptake of bilirubin, sulfobromophthalein, and other anions. Journal of Clinical Investigation, 48, 2156-2167.

Manghani, K. K., Lunzer, M. R., Billing, B. H., and Sherlock, S. (1975). Urinary and serum octopamine in patients with portal-systemic encephalopathy. Lancet, 2, 943-946.

Maxwell, J. D., Carrella, M., Parkes, J. D., Williams, R., Mould, G. P., and Curry, S. H. (1972). Plasma disappearance and cerebral effects of chlorpromazine in cirrhosis. Clinical Science, 43, 143-151.

Megyesi, C., Samols, E., and Marks, V. (1967). Glucose intolerance and diabetes in chronic liver disease. Lancet, 2, 1051-1056.

Morgan, M. H., Bolton, C. H., Morris, J. S., and Read, A. E. (1974). Medium chain triglycerides and hepatic encephalopathy. Gut, 15, 180-184.

Munro, H. N., Fernstrom, J. D., and Wurtman, R. J. (1975). Insulin, plasma aminoacid imbalance, and hepatic coma. Lancet, 1, 722-724.

Muto, Y., Takahashi, Y., and Kawamura, H. (1964). Effect of short chain fatty acids on the electrical activity of neo-, paleo- and archicortical systems. [Japanese.] Brain Nerve (Tokyo), 16, 608-611.

Nossel, H. L., Yudelman, I., Canfield, R. E., Butler, V. P., Jr., Spanondis, K., Wilner, G. D., and Qureshi, G. D. (1974). Measurement of fibrinopeptide A in human blood. Journal of Clinical Investigation, 54, 43-53.

Powell, L. W., and Axelsen, E. (1972). Corticosteroids in liver disease: Studies on the biological conversion of prednisone to prednisolone and plasma protein binding. Gut, 13, 690-696.

Preston, F. E., Holdsworth, C. D., and Malia, R. G. (1977). Proteins induced by vitamin $\mathrm{K}$ absence (PIVKA) in liver disease: response to IV vitamin $K_{1}$ therapy. (Abstract.) Gut, 18, A955.

Rake, M. O., Flute, P. T., Pannell, G., and Williams, R. (1970). Intravascular coagulation in acute hepatic necrosis. Lancet, 1, 533-537.

Read, A.'E., Sherlock, S., Laidlaw, J., and Walker, J. G. (1967). The Neuropsychiatric syndromes associated with chronic liver disease and an extensive portal-systemic collateral circulation. Quarterly Journal of Medicine, 36, 135-150.

Read, A. E., Laidlaw, J., and McCarthy, C. F. (1969).
Effects of chlorpromazine in patients with hepatic disease. British Medical Journal, 3, 497-499.

Rees, L. H., Besser, G. M., Jeffcoate, W. J., Goldie, D. J., and Marks, V. (1977). Alcohol-induced pseudo-Cushing's syndrome. Lancet, 1, 726-728.

Rosen, H. M., Yoshimura, N., Hodgman, J. M., and Fischer, J. E. (1977). Plasma amino acid patterns in hepatic encephalopathy of differing aetiology. Gastroenterology, 72, 483-487.

Rothschild, M. A., Oratz, M., Zimmon, D., Schreiber, S. S., Weiner, I., and Van Canegham, A. (1969). Albumin synthesis in cirrhotic subjects with ascites studied with carbonate ${ }^{14} \mathrm{C}$. Journal of Clinical Investigation, 48, 344-350.

Rubin, M. H., Weston, M. J., Bullock, G., Roberts, J., Langley, P. G., White, Y. S., and Williams, R. (1977). Abnormal platelet function and ultrastructure in fulminant hepatic failure. Quarterly Journal of Medicine, 46, 339-352.

Schaffner, S., and Popper, H. (1963). Capillarization of hepatic sinusoids in man. Gastroenterology, 44, 239-242.

Seligsohn, U., Shani, M., Ramot, B., Adam, A., and Sheba, C. (1970). Dubin-Johnson syndrome in Israel, II. Association with factor-VII deficiency. Quarterly Journal of Medicine, 39, 569-584.

Shear, L., Ching, S., and Gabuzda, G. J. (1970). Compartmentalization of ascites and edema in patients with hepatic cirrhosis. New England Journal of Medicine, 282, 13911396.

Sherwin, R., Joshi, P., Hendler, R., Felig, P., and Conn, H. O. (1974). Hyperglucagonemia in Laennec's cirrhosis. The role of portal systemic shunting. New England Journal of Medicine, 290, 239-242.

Smals, A. G., Kloppenborg, P. W., Njo, K. T., Knoben, J. M., and Ruland, C. M. (1976). Alcohol-induced Cushingoid syndrome. British Medical Journal, 2, 1298.

Thomas, D. P., Ream, J., and Stuart, R. K. (1967). Platelet aggregation in patients with Laennec's cirrhosis of the liver. New England Journal of Medicine, 276, 1344-1348.

Van Thiel, D. H., Lester, R., and Sherins, R. J. (1974). Hypogonadism in alcoholic liver disease, evidence for a double defect. Gastroenterology, 67, 1188-1199.

Vermeulen, A., Mussche, M., and Verdonck, L. (1973). Testosterone and oestradiol production rates and interconversions in normal males and male cirrhotics. Proceedings of the 4ih International Congress of Endocrinology, Washington, D.C., 1972, (International Congress Series, 273), Exc. Med. Cat. Abs. 305, p. 123. Excerpta Medica: Amsterdam.

Vlahcevic, Z. R., Buhac, I., Farrar, J. T., Bell, C. C. Jr., and Swell, L. (1971). Bile acid metabolism in patients with cirrhosis. I. Kinetic aspects of cholic acid metabolism. Gastroenterology, 60, 491-498.

Zieve, L. (1966). Hemolytic anemia in liver disease. Medicine (Balt.), 45, 497-505.

Zieve, L., and Nicoloff, D. M. (1974). Pathogenesis of hepatic coma. Annual Review of Medicine, 26, 143-157.

Zieve, L., and Olsen, R. L. (1977). Can hepatic coma be caused by a reduction of brain noradrenaline or dopamine? Gut, 18, 688-691. 